\title{
SAMir OKaSha, Agents and Goals in Evolution. Oxford: Oxford University Press, 2018, 254 pp., \$40.00
}

\author{
Adrian Stencel ${ }^{1}$
}

(C) Springer Nature Switzerland AG 2020

Samir Okasha's latest book focuses on the role of agency in evolutionary biology. Agents and Goals in Evolution is essential reading for philosophers and biologists interested in subjects where reference to agency occurs, including fitness optimization, kin selection, and social evolution. It also touches on the relation between rationality and evolution, which could make it of interest to scholars working outside evolutionary biology but seeking to understand the appeal to evolution in different scientific fields.

The main aim of the book is to provide a philosophical analysis of what Okasha calls 'agential thinking'. This involves treating biological units as rational agents with interests and goals that they try to accomplish by different means. The major consequence of agential thinking is the import of concepts that are supposed to explain human behaviour to the biological world-from bacteria to lions. This is accompanied by the use of intentional language, typical of human beings pursuing their desires. Biologists do not just say 'I want get a research grant', but also 'the bacteria that I study mutate because they want to grow faster'. This might sound inappropriate, but this way of thinking and speaking is pervasive in evolutionary biology. Agents and Goals in Evolution provides a great introduction to and analysis of the problem of agency at the intersection of philosophy and biology. It would be impossible to address all the issues raised by the book in this review, so I will briefly summarize the main parts of the book and then comment on some of the aspects that I found most stimulating.

The first part of the book constitutes an introduction to the world of agency in evolutionary biology. The author reviews the different concepts of agency and the various problems related to it, such as whether agency should be ascribed only to organisms or maybe also to other units, like genes or groups (such as social insects).

Adrian Stencel

adstencel@gmail.com

1 Faculty of Philosophy, Jagiellonian University, Kraków, Poland 
He introduces the concept of unity-of-purpose, which is supposed to clarify the cases when it makes sense to treat a given unit as an agent. But most importantly, in this part he introduces a distinction between agential thinking of type 1 and agential thinking of type 2. In Okasha's view, type 1 agential thinking occurs when we treat evolved entities as agents, whereas type 2 happens when we treat the process of natural selection as the agent itself. Okasha argues that the more useful sense of agential thinking is type 1 .

The second part of the book is focused on the controversial (but popular) idea that natural selection is an optimizing process that will tend to maximize fitness, which might be considered the goal that natural selection strives towards. A lot of issues related to the notion of agency are explored in this part of the book, including Hamilton's claim that organisms behave as if they want to maximize their inclusive fitness, and Fisher's 'Fundamental Theorem of natural selection'. This analysis leads Okasha to many interesting conclusions. The one that I found the most interesting is the idea that there is no general theoretical principle suggesting that natural selection will lead to the evolution of adaptations. Adaptationist claims must be scrutinized empirically, not theoretically. This philosophical position will, no doubt, lead to many discussions in the future.

The third part of the book focuses on analysing the relationship between evolutionary optimal behaviour and rational behaviour. One might distinguish here two main themes. First, the understanding of how rationality, understood as a phenotypic trait that characterises some organisms, evolved. And second, the examination of how agential thinking is used as a heuristic tool for understanding adaptive behaviour. Okasha tries to clarify the relationship between these two themes, putting forward the hypothesis that adaptive behaviour is a form of proto-rationality. This part of the book explores a wide arrange of interesting claims, including the suggestion that irrational behaviour can sometimes be an evolutionary optimal behaviour.

I found some Okasha's ideas especially illuminating. One of them is the concept of unity-of-purpose, which is supposed to explain the circumstances under which it is justified treating an entity as an agent, mainly when all its traits evolved to contribute to the same goal-enhancing the fitness of individual. This explains why biologists treat cats, for example, as agents. The reason is that cats exhibit a lot of traits that evolved to enhance their fitness, e.g., muscles that allow them to move, a digestive system that allow them to process food, and so on. Overall, cats seem to be well integrated and organized units made of elements that contribute to increasing their fitness. However, there are cases when it does not make sense to consider typical organisms as agents. For example, outlaw genes might break an organism's unity-of-purpose because they might promote their fitness enhancement (e.g. by distorting sex ratios in offspring as maternally inherited elements sometimes do) at the expense of the whole of which they are a part. In these cases, the organisms-as-agents heuristic might not be so appropriate. Fortunately, organisms contain mechanisms that eliminate what could be referred to as 'within agent agency', so treating them as agents will work in the majority of cases. Nevertheless, sometimes agency at different levels of the hierarchy might occur and this concept could be useful to spot its occurrence. One of the levels that would be interesting to explore under this framework is the level of the holobiont (a unit made of a host plus its symbiotic microbes), which some people consider to be bona fide individuals. Can holobionts be agents in evolution? 
One issue that is left unresolved is the relationship between agency and the units/levels of selection debate. Okasha writes (p. 43): "an obvious suggestion is that once we have identified the relevant level of selection/adaptation in any particular case, this will immediately yield the right candidate for the role of agent, if we wish to apply agential thinking". However, after stating that the units/levels of selection debate is complex, Okasha decides to take a different route in the book. Nevertheless, the question remains: what is the relationship between these two concepts? One obvious solution is to equate the two concepts. And I think that this makes some sense, as some biologists and philosophers (including this reviewer) have argued that to be a unit of selection a given entity must be composed of elements that share a 'common evolutionary fate'. In other words, an entity is a unit of selection if the elements that compose it depend on each other to the extent that they can increase their fitness only if they increase the fitness of the whole. As a result, in this view, a unit of selection would be an agent because it would fulfil the requirements of unity-of-purpose. However, not everyone would agree that those 'strict' requirements are necessary to be a unit of selection. If so, then the extension of those two concepts would be far more overlapping. Some units of selection might be agents, others might not. Whatever the proper answer is, this will definitely be an interesting topic to explore in the future.

The other idea that I found particularly appealing is Okasha's aforementioned conceptual distinction between agential thinking of type 1 and agential thinking of type 2 , as it removes an important source of potential confusion. Throughout the book Okasha offers arguments in favour of agential thinking 1, such as the fact organisms have evolved to do certain things (e.g. getting food, mating, etc.). They behave like agents and thus treating them in such a manner seems justified. The same, however, cannot be said about the agential thinking of type 2 which, as Okasha concludes, "works well if we confine attention to the simplest sorts of natural selection in which the selective environment is constant and the complexities of inheritance are ignored" (p. 230).

While I find this conceptual division and the arguments in favour of agential thinking of type 1 and against the type 2 persuasive, I am not convinced that agential thinking of type 2 should be limited to simple instances of natural selection. I think there is more room for this type of heuristic in evolutionary biology. For example, we might say that the goal of natural selection is to shift a frequency of phenotypes/genotypes to a different one in the next generation owing to fitness differences, but this may not always happen because of drift. Here natural selection seems goal-oriented while drift does not. In this way, thinking in terms of goals that natural selection pursues can help make clear the distinction between selection and drift. Even if I am wrong about this, the point I wish to emphasise is that evolutionary biology is perhaps broader than the analysis afforded by Okasha in his book. Thus, while his arguments are persuasive, they do not lead me to automatically remove agential thinking of type 2 from my 'conceptual toolbox'. Instead, it invites me to seek cases where this type of thinking might have a real value. And, for this, I am grateful to the author.

Publisher's Note Springer Nature remains neutral with regard to jurisdictional claims in published maps and institutional affiliations. 